\title{
CENTRO DE APRENDIZAJE TUMUL K'IN: EXPERIENCIA EDUCATIVA FORMAL. BELICE
}

Óscar Rubén Requenal

\section{Resumen}

El presente artículo surge de la ponencia que refería al Centro de Aprendizaje Tumul $\mathrm{K}$ 'in. En este centro, se desarrolla un programa para la juventud rural y, en especial, para la juventud desvalida maya que no ha tenido acceso a una educación secundaria y no ha continuado en la escuela convencional.

Palabras claves: multiculturalidad, cultura maya, educación formal.
Abstract

This article is the result of the paper Tumul K'in Learning Center. This center is a place where a program for rural youth, especially for Mayan people, is developed. The author describes how the program helps young people that have not had access to secondary education.

Keywords: multicultural, Maya culture, formal education.

$\mathrm{E}_{\mathrm{c}}^{\mathrm{l}}$

l Centro de Aprendizaje Tumul K'in se encuentra en la aldea de Blue Creek, distrito de Toledo, Belice. Es una institución que ofrece una educación secundaria no tradicional.

La historia de Tumul K'in inicia en 1997, cuando "U' Masewal kuxtal, el instituto maya de Belice", después de analizar la situación de los mayas, formó un comité para que desarrollara y promoviera el concepto de un centro educativo que respondiera a esa realidad, diera valor a la abundancia del conocimiento y sabiduría de los mayas, junto con un conocimiento moderno de la ciencia para ventaja de esta comunidad maya y de la humanidad.

El centro abrió oficialmente sus puertas a 12 estudiantes, el 31 de enero del 2002; hasta la fecha cuenta con 40 estudiantes, 34 hombres y 6 mujeres.

1 Asesor del Ministerio de Educación, Belice. Estudiante de la Maestria en Educación Rural.

Recibido: 30 de noviembre-2006 - Aprobado: 27 de febrero-2007 
Tiene un personal de 12 funcionarios: 1 director de manejo, 1 director académico, 3 profesores a tiempo completo, 2 se ofrecen voluntariamente, quienes enseñan y también administran los proyectos; el resto del personal trabaja en la granja y proporciona ayuda técnica. La escuela también cuenta con un equipo básico de agricultura, un laboratorio de cómputo con acceso a Internet, una granja pequeña con ganado y un sistema de rastreo de la naturaleza.

\section{Visión}

El Centro de Aprendizaje Tumul K'in será una organización reconocida nacional e internacionalmente, proporcionando la autorización, alternativa educativa y entrenando, al servicio basado en los valores de los mayas y la integración de la sabiduría moderna, conocimiento y tecnología a la juventud desvalida y a sus comunidades para conducir su desarrollo.

\section{Misión}

El Centro de Aprendizaje Tumul K'in proporciona la autorización, alternativa intercultural, para fijar la educación primaria a la juventud desvalida, desarrollar y compartir conocimientos y prácticas en las áreas de la utilización del suelo, la gerencia de recurso natural, creativa, experta, productiva, la agricultura orgánica, la tecnología sostenible y la educación con la fusión de la filosofia, del conocimiento y de la sabiduría tradicionales de los mayas con ciencia y tecnología modernas apropiadas.

\section{Metas fundamentales}

Desarrollar y promover un modelo de la actualización de las personas intercultural de la educación de los mayas que prepara personas críticas y reflexivas, capaces de hacer uso óptimo y sabio de sus recursos culturales, naturales y personales para su propio desarrollo y hacer líderes y modelos en el distrito de Toledo y Belice.

Funcionar un programa intercultural residencial de la educación de los mayas para la juventud desvalida de la edad de la escuela secundaria, basado en los valores y filosofia mayas e integración con ciencia y tecnología modernas.

Entregar los servicios educativos y de entrenamiento a la juventud y a los adultos que no califican para el programa residencial en un programa a 
distancia basado en los valores y filosofía e integración con ciencia y tecnología modernas apropiadas.

Investigar y desarrollar conocimientos y prácticas creativos en las áreas de la gerencia de recurso natural, agricultura, tecnología y educación sostenible con la integración del conocimiento y filosofía mayas con ciencia y tecnología modernas apropiadas.

Proporcionar servicios apropiados, técnicos en las comunidades mayas en el distrito de Toledo y en el país en las áreas de la agricultura sostenible, utilización del suelo, con espíritu emprendedor y una educación en las prácticas de gerencia del recurso natural.

\section{Estructura de organización}

La estructura organizativa del Centro de Aprendizaje Tumul K'in es la siguiente: cuenta con una Junta Directiva, que involucra a padres, organizaciones locales mayas, representantes de la comunidad y del negocio, el $\mathrm{Mi}$ nisterio de Educación y Agricultura. Tiene además, un Director de Manejo, un Director Académico, una Asociación de Profesores y Padres.

\section{Financiamiento}

Existen tres estrategias que posibilitan el financiamiento de la institución. Estas son: financiamiento de las agencias nacionales e internacionales, ayuda del Estado y el ingreso de la producción. En este sentido, todas las familias deben pagar el equivalente de E.U. \$75 en efectivo o lo equivalente en productos que ellas producen. Con este monto, se cubren los costos de alimento y de alojamiento en el centro.

\section{Política de la admisión}

La política de la admisión de Tumul K'in se basa en:

1. Conclusión de la escuela primaria (el énfasis no está en resultados de la examinación de la escuela primaria, según los requisitos de escuelas secundarias tradicionales).

2. Interés y disciplina.

3. Informe del alcalde de la comunidad donde vive el estudiante. 


\section{El programa residencial}

El programa sirve a la juventud rural y, particularmente, a la juventud desvalida maya que no ha tenido acceso a una educación secundaria y no ha continuado en la escuela convencional. Los y las estudiantes tienen una edad entre los 14 y los 16 años. Entre el 2003-2007, el énfasis será promover la participación de muchachas en la educación.

Una pista de dos gradas del estadio será ofrecida a los estudiantes para acomodar el horario del trabajo agrícola y la realidad económica de sus familias. Algunas familias, sin embargo, necesitan la ayuda de sus hijos para la labor agrícola. Estas tareas limitan la capacidad y la disponibilidad de sus hijos de estudiar a tiempo completo. Como respuesta, el calendario de la escuela se adapta a la actividad agrícola en que participan sus familias. La consideración, por lo tanto, se está dando para admitir estudiantes a tiempo completo, así como los estudiantes de medio tiempo que deben ayudar a sus familias.

Para hacer este horario dual, el centro ha desarrollado un sistema de evaluación que sirve para determinar las habilidades o capacidades mínimas de las y los estudiantes en cada campo de estudio. Así, quienes tienen un horario de medio tiempo, no tendrán que repetir los cursos en los cuales han demostrado capacidad en períodos anteriores del estudio. Este sistema también permitirá que estudiantes más avanzados se muevan más rápidamente con el programa.

Los y las estudiantes son socios en su educación. Son involucrados en el planeamiento y la operación de las actividades del centro para proporcionar el contexto de aprendizaje. Los métodos del centro en cuanto al plan de estudios y de la enseñanza proporcionan una metodología donde los estudiantes construyen sus propios conocimientos. Por ejemplo, si están involucrados en el manejo del presupuesto de alimento del centro, aprenden elementos de la nutrición y de contabilidad.

\section{Sostenibilidad}

Como parte integral de sus esfuerzos de ser autónomo y autosostenible, el centro utiliza lo que produce para alimentar a sus estudiantes y vende sus productos al mercado para recobrar ingresos. Tumul $\mathrm{K}$ 'in prevé que para el 2007, pueda generar un mínimo del $11 \%$ de sus requerimientos anuales. A este propósito, se unen las áreas de la producción de frutas, arroz, habas, 
maíz, cacao, pollos, patos, cabras, ovejas, cerdos, conejos, producción de la miel, piscicultura, agrosilvicultura y turismo. Actualmente, la producción y el proceso de la miel, el agro y las aves de corral y el ganado están contribuyendo al programa de sostenibilidad.

La educación ofrecida en el centro se funda en cinco principios dominantes que lo hacen especial:

1. Educación intercultural.

2. Comunidad de aprendizaje.

3. Integración del plan de estudios.

4. Integración de la teoría y de la práctica.

5. Independencia y espíritu emprendedor.

\section{Áreas de la especialización}

1. Proceso agrícola.

2. Agroproducción.

3. Uso sostenible de recursos naturales.

4. Turismo ecocultural.

El programa tiene una duración de cinco años: cuatro años en el campus y el quinto se enfoca en un proyecto emprendedor independiente.

¿Qué significa la educación intercultural?

- Plan de estudios/contenido.

- Práctica pedagógica.

- Cultura de la escuela.

- Participación de la comunidad.

\section{Práctica pedagógica}

- Planeamiento de colaboración.

- Diálogo.

- Integración del plan de estudios.

- Integración de la teoría y de la práctica.

- El aprender independiente y de colaboración.

- $\quad$ Profesores no tradicionales. 


\section{Cultura de la escuela}

- Valores mayas.

- Participación del estudiante.

- Democratización de la escuela.

- Trabajo de la comunidad.

- Conocimiento ambiental.

- Desarrollo personal con programa de la cosmovisión maya.

\section{Participación de la comunidad}

- Propiedad.

- Contribución de la comunidad.

- $\quad$ Participación del padre.

\section{Distribución del personal}

Dedican un total de 10 miembros del personal sobre todo para el programa residencial/académico; del total, 4 son voluntarios.

Califican académicamente pero carecen de profesores entrenados en la pedagogía. Son altamente motivados, aunque trabajan muchas horas y tienen una cantidad de trabajo pesado.

\section{Implicación parental}

- La asociación de los profesores y padres de Tumul $\mathrm{K}$ 'in fue formada en febrero del 2004.

- Hay una participación fuerte de la asociación de los profesores y padres.

- Los mismos padres son los que asisten a las reuniones.

- Esta es una preocupación importante de la asociación de los profesores y padres.

\section{Desafíos}

1. Desafios del programa residencial.

2. Alto índice de estudiantes que no terminan el programa.

3. La inscripción es baja.

4. Participación femenina pobre en el programa. 
5. Profesores: sueldos y entrenamiento.

6. Solamente algunos miembros del personal hablan Q'eqchi o Mopan

7. Mejorar el aspecto intercultural de la escuela.

\section{Discusión}

Los altos niveles de pobreza afectan a Belice. El estudio de la pobreza conducido en 1996 divulgó que el $25 \%$ de la población vive en pobreza y el $9,6 \%$, en pobreza extrema. De acuerdo con el reporte de 1999 sobre el desarrollo humano, la situación no ha mejorado mucho. La incidencia de la pobreza en el sector rural es del $42,3 \%$ y afecta particularmente a los mayas. Además, apunta a los niveles más altos del retraso del crecimiento, de la desnutrición y del analfabetismo (47,5\%). Son afectados por el acceso bajo a los servicios, esenciales para combatir esta pobreza. El informe humano del desarrollo, por ejemplo, observa que los programas y los servicios de desarrollo no están apuntando suficientemente a las áreas rurales, en particular a Toledo, donde están dos tercios de la población maya y recomienda la atención especial a este distrito.

Uno de los servicios, que es esencial para la reducción de la pobreza, es la educación. Para la gente maya, el problema de la educación es doble, no sólo se ve afectada por la pobre accesibilidad a la educación, sino por una carencia de la educación cultural pertinente y relevante. Mientras que el servicio educativo existente ofrece oportunidades importantes a la gente maya, también ha servido para erosionar los valores y el conocimiento cultural que podrían ser usados como base para la reducción de la pobreza.

En respuesta a la situación antes dicha, la experiencia educativa de Tumul $\mathrm{K}$ 'in es un modelo muy ambicioso y único que intenta desarrollar jóvenes rurales con una educación que sea sensible, ambientalmente amistoso y sostenible, equilibrado con teoría y experiencias prácticas.

Después de examinar el modelo de Tumul K'in, las siguientes son algunas de sus fortalezas.

La educación se ve como una herramienta que promueve a la humanidad para solidificar su propio conocimiento y aprecio cultural dentro del contexto de sus familias, de las comunidades y del mundo. La educación que reciben los preparará con el conocimiento, las habilidades, las actitudes y los valores que les ayudarán a reconocer que juegan un papel muy importante en el desarrollo de sus comunidades.

El enfoque de los programas se basa en los siguientes valores, que son planteados por la Conferencia Mundial de Jomtien: 
La correlación de la educación con el trabajo. Este énfasis es visible en el desarrollo curricular y programas ejecutados, donde los estudiantes ponen en práctica la teoría que aprenden. Las habilidades de la vida se integran en el plan de estudios, que incluyen: dirección, interdependencia (los estudiantes lavan y cocinan).

Se valora el ambiente, la cultura, la familia, los derechos humanos y la equidad de género. Es interesante observar que durante la II Reunión de Ministros de Educación de las Américas, celebrada en Uruguay en el 2001, se identificaron los valores ya mencionados, como la base para una educación sostenible especialmente para el área rural. De manera que la institución dará énfasis en promover la participación de muchachas del área rural en la educación. Esta es una meta que debe ser resaltada y reconocida como un logro importante para el género femenino. Se debe apuntar que en muchas comunidades mayas, la educación de la mujer no se ha valorado como debe ser, porque la educación tradicional se ve descontextualizada de la cultura y valores de la comunidad.

Con respecto a la cultura, Tumul $\mathrm{K}$ 'in se debe reconocer como el pionero en este tema. La cultura maya es integrada en los programas de estudio y se viven las experiencias diarias. Por ejemplo, hay actividades culturales, las reuniones de padres se llevan a cabo en el idioma maya. Según la conferencia de París en 1985, la alfabetización en lengua matemal refuerza la identidad y la herencia cultural, por lo que se asume como una política "alfabetizar a los niños y a las niñas en su propia lengua".

Las prácticas agrícolas también demuestran que están sensibilizando a los estudiantes y que aprecian la interdependencia que existe entre el ambiente y la humanidad: lo que se toma de la naturaleza se debe devolver a la naturaleza. Este concepto implica un equilibrio cuidadoso y armonioso de intercambios ambientales. Los elementos y los procedimientos naturales y beneficiosos se utilizan en la producción agrícola y el proceso. Los fertilizantes naturales, orgánicos, herbicidas, pesticidas se usan en la agricultura para ver cuál está en la naturaleza, con el fin de preservar y proteger el ambiente y al ser humano. Las prácticas como agrosilvicultura y cultivo mezclado respetan la armonía de la naturaleza, así como protegen la tierra y el ambiente. Lo dicho está de acuerdo con el Foro de Jomtien (1990), que propone una política de educación sostenible con el ambiente.

Otro éxito del modelo Tumul $\mathrm{K}$ 'in que pienso se debe mencionar es su política de la disciplina. La escuela tiene un consejo disciplinario compuesto 
por igual cantidad de estudiantes y profesores. Cuando un estudiante tiene problemas disciplinarios, el consejo convoca al estudiante. La sesión se centra en el desarrollo personal más que en el castigo. Esto debe ser elogiado, ya que ayuda a promover el pilar de aprender a vivir juntos, propuesto por la UNESCO. Se debe observar que este acercamiento concuerda con las propuestas hechas por los Ministros de Educación en Dakar en el año 2000.

\section{Desafíos}

Mientras que el centro está haciendo grandes logros en educar a nuestra gente joven rural, hay algunas áreas que necesitan mejoría, para lograr las metas planteadas. Estas incluyen:

1. La escuela todavía no es autosostenible. Los programas siempre dependen del financiamiento externo.

2. Hay un alto número de estudiantes que han dejado las clases, porque en algunos casos los padres necesitan que trabajen en la granja. Las expectativas de la escuela, en ciertas ocasiones, no están en línea con la de los estudiantes (la disciplina, el trabajo, la ética). Algunos estudiantes desean un programa rápido y no quieren pasar cinco años en una institución.

3. Hay necesidad de estructurar un programa de entrenamiento en pedagogía para los profesores.

4. Hay necesidad de establecer una política clara para emplear los profesores; asimismo, contar con un sistema que les proporcione incentivos y remuneración. Actualmente, el incentivo es la satisfacción profesional basada en la motivación y el interés.

5. Se debe elaborar un sistema sistemático de valoración, supervisión y evaluación de los profesores. 


\section{REFERENCIAS}

Center of Learing. (2006). TUMULK'IN. Recuperado en: http://www.geocities. com/mayacomanage/tumulkin.htm.

Maya Leaders' Alliance (MLA). (2006). The struggle to preserve maya land. Recuperado en: http://www.geocities.com/mayacomanage/mla.htm. 